\title{
La ecografía es un método aceptable para el diagnóstico de patologías subacromiales
}

\author{
Ultrasound is an acceptable test for the diagnosis of subacromial disorders
}

\section{Objetivo}

Evaluar la precisión diagnóstica de la ecografía para detectar patologías subacromiales en pacientes que consultan a centros de primer y segundo nivel de atención.

\section{Diseño}

Revisión sistemática y meta-análisis. Este trabajo actualizó la búsqueda de una revisión previa (1985-2001) cumpliendo con las recomendaciones de la Colaboración Cochrane. Dos revisores independientes buscaron en PubMed y Embase artículos adicionales publicados entre 2001 y 2010. Se incluyó una búsqueda manual de las referencias de los estudios.

\section{Lugar}

Holanda.

\section{Pacientes}

Adultos con sospecha de lesiones subacromiales: bursitis subacromial, tendinopatías del manguito rotador, tendinitis calcificante, rotura parcial o total.

\section{Descripción de las pruebas y del gold standard}

Este estudio toma como gold standard o test de referencia* a la resonancia magnética nuclear, cirugía y/o radiografía.

\section{Medición de resultados principales}

Se incluyeron 23 estudios para el análisis principal. Se evaluó la sensibilidad* y especificidad* de la ecografía.

\section{Resultados principales}

Ninguno de los estudios se desarrollo en un centro de atención primaria. Se analizaron 23 estudios: 22 reportaron ruptura completa del manguito rotador, 15 lesión parcial, 3 bursitis subacro-
Ottenheijm RP y col. Arch Phys Med Rehabil. Oct 2010; 91:1616-25.

mial, 2 tendinopatía y 2 tendinitis calcificante.

La tabla 1 resume la sensibilidad y especificidad de la ecografía en el diagnóstico de ruptura total y parcial del manguito rotador. Para las otras patologías no fue posible realizar la estimación de resultados ponderados. Para bursitis subacromial la sensibilidad osciló entre 0,79 y 0,81, y la especificidad entre 0,94 a 0,98 . Para la tendinopatía, la sensibilidad osciló entre 0,67 y 0,93 , y la especificidad entre 0,88 y 1,0 . La sensibilidad para la tendinitis calcificada fue de 1,0 en ambos estudios, y la especificidad osciló entre 0,85 y 0,98.

Tabla1: Características operativas de la ecografía para el diagnóstico de patología subacromial.

\begin{tabular}{l|c|c}
\multicolumn{1}{c|}{ Patologia } & \multicolumn{1}{c|}{$\begin{array}{c}\text { Sensibilidad } \\
(\mathbf{I C 9 5 \% )}\end{array}$} & $\begin{array}{c}\text { Especificidad } \\
(\mathbf{I C 9 5} \%)\end{array}$ \\
\hline $\begin{array}{l}\text { Rotura total del manguito } \\
\text { rotador }\end{array}$ & $0,95(0,90$ a 0,97$)$ & $0,96(0,93$ a 0,96) \\
\hline $\begin{array}{l}\text { Rotura parcial del } \\
\text { manguito rotador }\end{array}$ & $0,72(0,58$ a 0,83$)$ & $0,93(0,89$ a 0,96) \\
\hline
\end{tabular}

\section{Conclusiones}

La ecografía es un método aceptable para el diagnóstico de patologías de hombro donde el tratamiento conservador fracasó, para diagnosticar o descartar rupturas totales y diagnosticar rupturas parciales, y en menor medida diagnosticar tendinopatias, bursitis subacromial y tendinitis calcificante ${ }^{1}$. La precisión del diagnóstico ecográfico en problemas agudos aun no se determinó. Para las otras lesiones no fue suficiente el poder estadístico de este estudio.

Fuente de financiamiento: no referida. Ningún autor declara conflicto de intereses.

\section{Comentario}

Todavía no hay consenso acerca del método más preciso para el diagnóstico de problemas subacromiales. Estas patologías cuentan con un componente anatomopatológico y uno dinámico. La evaluación debe basarse en la clínica y capacidad funcional de paciente.

La combinación de la historia clínica, el examen físico y la ecografía son suficientes para un diagnóstico certero y permite al médico de atención primaria dirigir un tratamiento adecuado al paciente en la mayoría de los casos. La ecografía permite separar las aguas entre los pacientes que requieren cirugía y los que no la precisan.

\section{Conlusiones del comentador}

La ecografía, muy difundida en la evaluación de estas patologías, es una prueba diagnóstico con adecuadas características operativas. Cabe mencionar que ninguno de los estudios de la presente revisión sistemática fue realizado en un ámbito de atención primaria, donde este método diagnóstico es muy utilizado.

Denise Cytryn [ Servicio de Medicina Familiar y Comunitaria del Hospital Italiano de Buenos Aires. denise.cytryn@hospitalitaliano.org.ar ]

Cytryn D. La ecografía fue un método aceptable para el diagnostico de patologías subacromiales. Evid Act Pract Ambul Abr-Jun 2012;15(2):52. Comentado de: Ottenheijm RP; Jansen MJ; Staal JB y col. Accuracy of diagnostic ultrasound in patients with suspected subacromial disorders: A systematic review and meta-analysis. Arch Phys Med Rehabil. Oct 2010; 91:1616-25.

Referencias

1. Anderson BC, Anderson RJ. Evaluation of the patient with shoulder complaints. UpToDate 2012. 